\title{
Legendre Polynomials Iterative Technique for Solving a Class of Nonlinear Optimal Control Problems
}

\author{
Hussein Jaddu and Amjad Majdalawi \\ Electronics Engineering Department, Faculty of Engineering, Al Quds University, \\ Jerusalem, Palestine \\ jaddu@eng.alquds.edu,amjadmajdalawi@hotmail.com
}

\begin{abstract}
A computational algorithm is proposed to solve a class of nonlinear optimal control problems. The proposed algorithm is based on replacing the original nonlinear optimal control problem by a sequence of time-varying linear quadratic optimal control problems. This is accomplished by employing an iterative technique developed by Banks [1-5] which is based on replacing the original nonlinear system by a sequence of linear time-varying systems. Then each of the time-varying linear quadratic optimal control problems is transformed into a standard quadratic programming problem by parameterizing the state variables by a finite length Legendre polynomials with unknown parameters. The solution of a standard nonlinear optimal control problem is presented, to show the effectiveness of the proposed method.
\end{abstract}

Keywords: Nonlinear optimal control problem, Banks Iterative Technique, Legendre polynomials, State parameterization

\section{Introduction}

One of the approaches to solve the optimal control problems is to use the direct methods. These methods are based on converting the dynamic optimal control problem into a nonlinear programming problem [6-15]. The direct methods are implemented by either discretization [6-9] or by parameterization which in turn can be implemented by parameterizing the state variables [10-12], control variables [13] or both the state and control variables [14,15].

In [10-12], Jaddu proposed methods using the quasilinearization technique to convert the nonlinear optimal control problems into a sequence of time-varying linear quadratic optimal control problems, then he applied the state parameterization using Chebyshev polynomials to solve the time-varying LQ optimal control problems. The idea of quasilinearization [16] is to expand the performance index up to the second order and the constraints up to the first order around a nominal state and control vectors. Recently in [1-5] Banks and his coauthors developed a method to treat the nonlinear systems by means of iterative approach.

In this paper, we present a method that is based on the Banks iterative technique to replace a class nonlinear optimal control problem by a sequence of time-varying linear quadratic optimal control problems. Then each of these problems is converted into a quadratic programming problem by state parameterization using Legendre polynomials. The method in this paper is classified as a direct method. 


\section{Problem Statement}

The optimal control problem treated in this paper can be stated as follows: Find an optimal controller $u^{*}(t)$ that minimizes the following performance index

$$
J=\int_{0}^{t_{f}}\left(x^{T} Q x+u^{T} R u\right) d t
$$

subject to the system state equations and initial conditions

$$
\dot{x}=A(x) x+B(x) u \quad x(0)=x_{0}
$$

where $Q$ is a positive semidefinite matrix, $R$ is a positive definite matrix, $x \in R^{n}$ is the state vector, $u \in R^{m}$ is the control vector, $x_{0} \in R^{n}$ is the initial condition vector. We will assume that: $m \leq n$, and $t_{f}$ is fixed.

The proposed algorithm to solve the mentioned problem consists of the following steps:

1. The first step, is to convert the nonlinear quadratic optimal control problem into a sequence of time-varying linear quadratic optimal control problems by using Banks method.

2. The second step is to convert each of these problems into quadratic programming problem by using the Legendre polynomials to parameterize the state variables.

3. The third step is to solve the quadratic programming problem. Then the solution of the state and the control variables are used in the first step for new iteration.

\section{Iterative Method and Problem Reformulation}

The iterative technique which was developed by Banks and his coauthors [1-5], is employed in this paper to convert the nonlinear optimal control problem (1)- (2) into a sequence of time-varying linear quadratic optimal control problems as follows:

for $i \geq 1$

Minimize

$$
J^{[i]}=\int_{0}^{t_{f}}\left(x^{[i]^{T}} Q x^{[i]}+u^{[i]^{T}} R u^{[i]}\right) d t
$$

subject to the linearized state equations and initial conditions

$$
\begin{aligned}
& \dot{x}^{[i]}=A\left(x^{[i-1]}(t)\right) x^{[i]}+B\left(x^{[i-1]}(t)\right) u^{[i]}, x^{[i]}(0)=x_{0} \\
& \text { and } x^{[0]}(t)=x_{0}
\end{aligned}
$$

Each of the time-varying linear quadratic optimal control problems (3)-(4) is converted into a quadratic programming problem by applying the state parameterization using Legendre polynomials. And since Legendre polynomials are orthogonal on the interval $\tau \in[-1,1]$, it is necessary to transform the time interval of the original problem $t \in\left[0, t_{f}\right]$ into $\tau \in[-1,1]$. This will transform (3)-(4) into the problem: for $i \geq 1$ Minimize 


$$
J^{[i]}=\frac{t_{f}}{2} \int_{-1}^{1}\left(x^{[i]^{T}} Q x^{[i]}+u^{[i]^{T}} R u^{[i]}\right) d \tau
$$

subject to:

$$
\frac{d x}{d \tau}^{[i]}=\frac{t_{f}}{2}\left[A\left(x^{[i-1]}(\tau)\right) x^{[i]}+B\left(x^{[i-1]}(\tau)\right) u^{[i]}\right], x^{[i]}(-1)=x_{0}
$$

and $x^{[0]}(t)=x_{0}$.

\section{State Parameterization via Legendre Polynomials}

\subsection{System State Equation Approximation}

For a detailed description of how to apply the state parameterization technique see [10-12]. To convert the optimal control problem (5)-(6) into a quadratic programming problem, some state variables are approximated by a finite length Legendre series with unknown parameters. Then, the remaining state and control variables are determined as a function of the unknown parameters of the state variables from the state equations (6). The initial conditions of the system are replaced by equality constraints. These approximated state and control variables are then directly substituted into the system performance index.

Applying the method described in [10-12], the state variables can be approximated by using Legendre polynomials of the first kind as follows

$$
x_{j}=\sum_{i=0}^{N} a_{i}^{(j)} P_{i}(\tau) \quad j=1,2, \ldots, n
$$

where $N$ is the length of the approximated series which depends on the required accuracy; large values of $N$ yield good accuracy at the expense of computational time and vice versa, $a_{i}$ are the unknown parameters and $P_{i}(\tau)$ is $\mathrm{i}^{\text {th }}$ order first kind Legendre polynomial. The control variables are obtained from the system state equations as a function of the state variables unknown parameters. These control variables can be written in terms of a finite length series of Legendre polynomials with unknown parameters $b_{i}$ as follows

$$
u_{l}=\sum_{i=0}^{N} b_{i}^{(l)} P_{i}(\tau) \quad l=1,2, \ldots, m
$$

where the unknown parameters $b_{i}^{(l)}$ are function of the state variables unknown parameters $a_{i}^{(j)}$.

Also, in state parameterization, it is necessary to determine the derivative of the state variables, which can be obtained as

$$
\dot{x}_{j}=\sum_{i=0}^{N} a_{i}^{(j)} \dot{P}_{i}(\tau) \quad j=1,2, \ldots, n
$$

The derivative of Legendre polynomials can be obtained by the following recursive formula [17],

$$
\dot{P}_{i+1}(\tau)=(2 i+1) P_{i}(\tau)+\dot{P}_{i-1}(\tau)
$$


where $\dot{P}_{0}(\tau)=0$ and $\dot{P}_{1}(\tau)=P_{0}(\tau)$. From this recursive formula it is possible to obtain $\dot{\boldsymbol{P}}(\tau)$ as follows,

$$
\dot{\boldsymbol{P}}(\tau)=\boldsymbol{D} \boldsymbol{P}(\tau)
$$

where the matrix $\boldsymbol{D}$ is called the differentiation operational matrix of Legendre polynomials and is given by

$$
D=\left[\begin{array}{ccccccccc}
0 & 0 & 0 & 0 & 0 & 0 & 0 & 0 & \ldots \\
1 & 0 & 0 & 0 & 0 & 0 & 0 & 0 & \ldots \\
0 & 3 & 0 & 0 & 0 & 0 & 0 & 0 & \ldots \\
1 & 0 & 5 & 0 & 0 & 0 & 0 & 0 & \ldots \\
0 & 3 & 0 & 7 & 0 & 0 & 0 & 0 & \ldots \\
1 & 0 & 5 & 0 & 9 & 0 & 0 & 0 & \ldots \\
0 & 3 & 0 & 7 & 0 & 11 & 0 & 0 & \ldots \\
1 & 0 & 5 & 0 & 9 & 0 & 13 & 0 & \ldots \\
\vdots & \vdots & \vdots & \vdots & \vdots & \vdots & \vdots & \ddots & \ddots
\end{array}\right]
$$

\subsection{Time-Varying Matrices $A\left(x^{[i-1]}(\tau)\right)$ and $B\left(x^{[i-1]}(\tau)\right)$ Approximation}

Equation (6) shows that the two matrices $A\left(x^{[i-1]}(\tau)\right)$ and $B\left(x^{[i-1]}(\tau)\right)$ are a function of $\tau$, therefore it is necessary to express every $\tau$ dependant element in both matrices in terms of a Legendre series of known parameters. To this end, let $A_{j l}(\tau)=g\left(x^{[i-1]}(\tau), \tau\right)$ be the $(j, l)$ element of the matrix $A\left(x^{[i-1]}(\tau)\right)$ where $x^{[i-1]}(\tau)$ is the nominal trajectory of the previous iteration. Then the term $A_{j l}(\tau)$ can be expressed in terms of a Legendre series of known parameters of the form

$$
A_{j l}(\tau)=\sum_{i=0}^{M} W_{i} P_{i}(\tau)
$$

where the coefficients $W_{i}$ are given by [17]

$$
W_{i}=\frac{2 i+1}{2} \int_{-1}^{1} g(\tau) P_{i}(\tau) d \tau
$$

The same approximation can be done for the matrix $B\left(x^{[i-1]}(\tau)\right)$.

Equation (6) also shows that $A\left(x^{[\mathrm{i}-1]}(\tau)\right), B\left(x^{[\mathrm{i}-1]}(\tau)\right)$ are time-varying matrices expressed as a function of Legendre polynomials of the previous iteration, and since the matrix $A\left(x^{[i-1]}(\tau)\right)$ is multiplied by $x^{[i]}$, which is expressed in terms of Legendre series with unknown parameters, and the matrix $B\left(x^{[i-1]}(\tau)\right)$ is multiplied by $u^{[i]}$, which is also expressed as a function of Legendre polynomials with unknown parameters, it is necessary to have a multiplication algorithm to multiply Legendre series. This algorithm is given by the following results:

Given two Legendre series

$$
X=\sum_{i=0}^{n} x_{i} P_{i}
$$




$$
Y=\sum_{j=0}^{m} y_{j} P_{j}
$$

Then the multiplication of these two Legendre series is a Legendre series of length $n+m$ given by

$$
X . Y=\sum_{i=0}^{n} \sum_{j=0}^{m} x_{i} y_{j} P_{i} P_{j}
$$

where $P_{i} P_{j}$ is given by [18]

$$
P_{i} P_{j}=\sum_{r=0}^{j} \frac{A_{r} A_{i-r} A_{j-r}}{A_{i+j-r}} \frac{2 i+2 j-4 r+1}{2 i+2 j-2 r+1} P_{i+j-2 r}
$$

where $i \geq j, A_{r}=\frac{(2 r-1) ! !}{r !}, \mathrm{A}_{0}=1$ and $! !$ is the double factorial.

\subsection{Initial State Constraints Approximation}

The next step is to approximate the initial condition vector $x(-1)=x_{0}$ by substituting $\tau=-1$ into (7) and using the following Legendre initial value property [17]

$$
P_{n}(-1)=(-1)^{n}
$$

the initial condition vector can be approximated as follows

$$
a_{0}^{(j)}-a_{1}^{(j)}+a_{2}^{(j)}-a_{3}^{(j)}+\cdots+(-1)^{N} a_{N}^{(j)}-x_{k}(-1)=0 \quad j=1,2, \ldots, n
$$

where $x_{k}(-1)=x_{0}$.

\subsection{Performance Index Approximation}

The last step is to approximate the performance index $I$. To do this, (7) and (8) are rewritten in matrix form as follows

$$
\begin{gathered}
{\left[\begin{array}{c}
\mathrm{x}_{1}^{[i]} \\
\mathrm{x}_{2}^{[\mathrm{i}]} \\
\vdots \\
\mathrm{x}_{\mathrm{n}}^{[\mathrm{i}]}
\end{array}\right]=\left[\begin{array}{cccc}
\mathrm{a}_{0}^{(1)} & \mathrm{a}_{1}^{(1)} & \ldots & \mathrm{a}_{\mathrm{N}}^{(1)} \\
\mathrm{a}_{0}^{(2)} & \mathrm{a}_{1}^{(2)} & \ldots & \mathrm{a}_{\mathrm{N}}^{(2)} \\
\vdots & \vdots & \vdots & \vdots \\
\mathrm{a}_{0}^{(\mathrm{n})} & \mathrm{a}_{1}^{(\mathrm{n})} & \ldots & \mathrm{a}_{\mathrm{N}}^{(\mathrm{n})}
\end{array}\right]\left[\begin{array}{c}
\mathrm{P}_{0} \\
\mathrm{P}_{1} \\
\vdots \\
\mathrm{P}_{\mathrm{N}}
\end{array}\right]} \\
{\left[\begin{array}{c}
\mathrm{u}_{1}^{[\mathrm{i}]} \\
\mathrm{u}_{2}^{[\mathrm{i}]} \\
\vdots \\
u_{\mathrm{m}}^{[\mathrm{i}]}
\end{array}\right]=\left[\begin{array}{cccc}
\mathrm{b}_{0}^{(1)} & \mathrm{b}_{1}^{(1)} & \ldots & \mathrm{b}_{\mathrm{N}}^{(1)} \\
\mathrm{b}_{0}^{(2)} & \mathrm{b}_{1}^{(2)} & \ldots & \mathrm{b}_{\mathrm{N}}^{(2)} \\
\vdots & \vdots & \vdots & \vdots \\
b_{0}^{(\mathrm{m})} & \mathrm{b}_{1}^{(\mathrm{m})} & \ldots & \mathrm{b}_{\mathrm{N}}^{(\mathrm{m})}
\end{array}\right]\left[\begin{array}{c}
\mathrm{P}_{0} \\
\mathrm{P}_{1} \\
\vdots \\
\mathrm{P}_{\mathrm{N}}
\end{array}\right]}
\end{gathered}
$$

or in compact form 


$$
x^{[i]}=L_{1} P \quad u^{[i]}=L_{2} P
$$

Substituting (22) into (5)

$$
\hat{\mathrm{J}}^{[\mathrm{i}]}=\frac{\mathrm{t}_{\mathrm{f}}}{2} \int_{-1}^{1}\left(\mathrm{P}^{\mathrm{T}} \mathrm{L}_{1}{ }^{\mathrm{T}} \mathrm{QL} \mathrm{L}_{1} \mathrm{P}+\mathrm{P}^{\mathrm{T}} \mathrm{L}_{2}{ }^{\mathrm{T}} \mathrm{RL}_{2} \mathrm{P}\right) \mathrm{d} \tau
$$

Where $\hat{J}^{[i]}$ is the approximate value of $J^{[i]}$.

The value of the approximated performance index in (23) can be given as follows:

Theorem 1 The value of the approximated performance index $\hat{I}$ given in (23) is given by

$$
\hat{I}=\frac{t_{f}}{2} \sum_{i=1}^{N+1} \frac{2}{2 i-1}\left(m_{i i}+z_{i i}\right)
$$

or

$$
\hat{I}=\frac{t_{f}}{2}(\operatorname{trace}(M)+\operatorname{trace}(Z)) \sum_{i=1}^{N+1} \frac{2}{2 i-1}
$$

where $m_{i i}$ and $z_{i i}$ are the diagonal elements of the symmetrical matrices $M=L_{1}{ }^{T} Q L_{1}$ and $Z=L_{2}{ }^{T} R L_{2}$ respectively.

Proof: Following the procedure used in [10] to prove similar result for Chebyshev polynomials, equation (23) can be rewritten as

$$
\hat{l}=\frac{t_{f}}{2} \int_{-1}^{1}\left(P^{T} M P+P^{T} Z P\right) d \tau
$$

The term $P^{T} M P$ can be expanded into

$$
\begin{array}{r}
P^{T} M P=m_{11} P_{0} P_{0}+2 m_{12} P_{0} P_{1}+2 m_{13} P_{0} P_{2}+\cdots+2 m_{1, N+1} P_{0} P_{N} \\
+m_{22} P_{1} P_{1}+2 m_{23} P_{1} P_{2}+\cdots+2 m_{2, N+1} P_{1} P_{N} \\
+m_{33} P_{2} P_{2}+\cdots+2 m_{3, N+1} P_{2} P_{N} \\
\vdots \\
+m_{N+1, N+1} P_{N} P_{N}
\end{array}
$$

Using the integration property of Legendre polynomials [17] ,

$$
\int_{-1}^{1} P_{n}(\tau) P_{m}(\tau) d \tau= \begin{cases}0 & n \neq m \\ \frac{2}{2 n+1} & n=m\end{cases}
$$

The integration of all terms in (27) $P_{n} P_{m}$, when $m \neq n$ is zero. The remaining parts of (27) is given by

$$
P^{T} M P=m_{11} P_{0} P_{0}+m_{22} P_{1} P_{1}+m_{33} P_{2} P_{2}+\cdots+m_{N+1, N+1} P_{N} P_{N}
$$

which can be integrated as follows: 
$\int_{-1}^{1}\left(m_{11} P_{0} P_{0}+m_{22} P_{1} P_{1}+m_{33} P_{2} P_{2}+\cdots+m_{N+1, N+1} P_{N} P_{N}\right) d \tau=\sum_{i=1}^{N+1} m_{i i} \int_{-1}^{1} P_{i-1} P_{i-1} d \tau$

Hence,

$$
\sum_{\mathrm{i}=1}^{\mathrm{N}+1} \mathrm{~m}_{\mathrm{ii}} \int_{-1}^{1} \mathrm{P}_{\mathrm{i}-1} \mathrm{P}_{\mathrm{i}-1} \mathrm{~d} \tau=\sum_{\mathrm{i}=1}^{\mathrm{N}+1} \mathrm{~m}_{\mathrm{ii}} \frac{2}{2 \mathrm{i}-1}=\operatorname{trace}(\mathrm{M}) \sum_{\mathrm{i}=1}^{\mathrm{N}+1} \frac{2}{2 \mathrm{i}-1}
$$

This gives proof of the first part of the previous theorem. Following the same procedure, integration of the second part $P^{T} Z P$ can be computed.

The performance index in (23) or (25) can be rewritten as follows

$$
\hat{J}=\frac{1}{2} a^{T} H a
$$

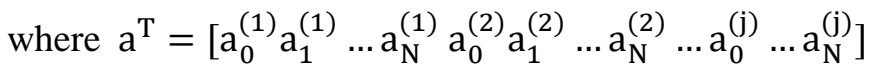

is the unknown parameter vector and $H$ is a positive definite Hessian matrix [10] given by ,

$$
H=\frac{\partial^{2} \hat{f}}{\partial a_{i}^{(k)} \partial a_{j}^{(k)}}
$$

where $i_{y} j=0,1, \ldots, N$ and $k=1,2, \ldots, N$

The original nonlinear quadratic optimal control problem can now be restated as follows:

$$
\min _{a} \frac{1}{2} a^{T} H a
$$

subject to

$$
\mathrm{F} a=\mathrm{b}
$$

where the equality constraints are due to initial conditions and in some cases unsatisfied state equations.

The standard quadratic programming problem (33)-(34) can be solved by matrix-vector multiplication as follows:

$$
a=H^{-1} F^{T}\left(F H^{-1} F^{T}\right)^{-1} b
$$

To solve the original nonlinear optimal control problem (1)-(2), we need to solve timevarying linear quadratic optimal control problem (5)-(6) iteratively using the proposed method until

$$
\left|\hat{J}^{[i+1]}-\hat{J}^{[i]}\right|<\epsilon
$$

is satisfied. In this work we consider $\epsilon=10^{-5}$. 


\section{Computation Results}

\section{Van der Pol Oscillator}

Find an optimal controller $u^{*}(t)$ that minimizes the following performance index

$$
I=\frac{1}{2} \int_{0}^{5}\left(x_{1}^{2}+x_{2}^{2}+u^{2}\right) d t
$$

subject to

$$
\begin{aligned}
& \dot{x}_{1}=x_{2} \quad, x_{1}(0)=1 \\
& \dot{x}_{2}=-x_{1}+\left(1-x_{1}^{2}\right) x_{2}+u \quad, x_{2}(0)=0
\end{aligned}
$$

Using the technique in section 3 , the problem can be reformulated as: For $i \geq 1$, Find $u^{[i]}$ that minimize

$$
f^{[i]}=\frac{1}{2} \int_{0}^{5}\left(\left(x_{1}^{[i]}\right)^{2}+\left(x_{2}^{[i]}\right)^{2}+\left(u^{[i]}\right)^{2}\right) d t
$$

subject to

$$
\begin{aligned}
& \dot{x}_{1}{ }^{[i]}=x_{2}{ }^{[i]} \quad, x_{1}{ }^{[i]}(0)=1 \\
& \left.\dot{x}_{2}{ }^{[i]}=-x_{1}{ }^{[i]}\right]+\left(1-\left(x_{1}^{[i-1]}\right)^{2}\right) x_{2}{ }^{[i]}+u^{[i]]}, x_{2}{ }^{[i]}(0)=0
\end{aligned}
$$

After changing the time interval $t \in[0,5]$ to the interval $\tau \in[-1,1]$,we get

$$
J^{[i]}=\frac{5}{4} \int_{-1}^{1}\left(\left(x_{1}^{[i]}\right)^{2}+\left(x_{2}^{[i]}\right)^{2}+\left(u^{[i]}\right)^{2}\right) d \tau
$$

subject to

$$
\begin{aligned}
& \dot{x}_{1}^{[i]}=\frac{5}{2} x_{2}{ }^{[i]} \quad, x_{1}{ }^{[i]}(-1)=1 \\
& \dot{x}_{2}{ }^{[i]}=\frac{5}{2}\left(-x_{1}{ }^{[i]}+\left(1-\left(x_{1}^{[i-1]}\right)^{2}\right) x_{2}{ }^{[i]}+u^{[i]}\right), x_{2}{ }^{[i]}(-1)=0
\end{aligned}
$$

This problem is solved by approximated $x_{1}(\tau)$ by 9 th order Legendre series and by 15 th order Legendre series. $x_{2}(\tau)$ is determined from (44) while $u(\tau)$ is determined from (45). Table 1 shows the optimal values of the cost function $I$ versus iteration $i$ for Legendre polynomial with $N=9$ and $N=15$. Table 2 shows the optimal values $I$ obtained using some other methods for comparison purposes. Figure 1 shows the optimal trajectories of the Van der Pol oscillator problem. 
Table 1. Optimal values of $\hat{J}^{[i]}$

\begin{tabular}{|c|c|c|}
\hline iteration $\mathrm{i}$ & $\mathrm{N}=9$ & $\mathrm{~N}=15$ \\
\hline 0 & 0.9533638119 & 0.9533622622 \\
\hline 1 & 1.4515573659 & 1.4515286441 \\
\hline 2 & 1.4496686129 & 1.4496983487 \\
\hline 3 & 1.4493656156 & 1.4493288574 \\
\hline 4 & 1.4494054733 & 1.4494048523 \\
\hline 5 & 1.4494004218 & 1.4493960944 \\
\hline
\end{tabular}

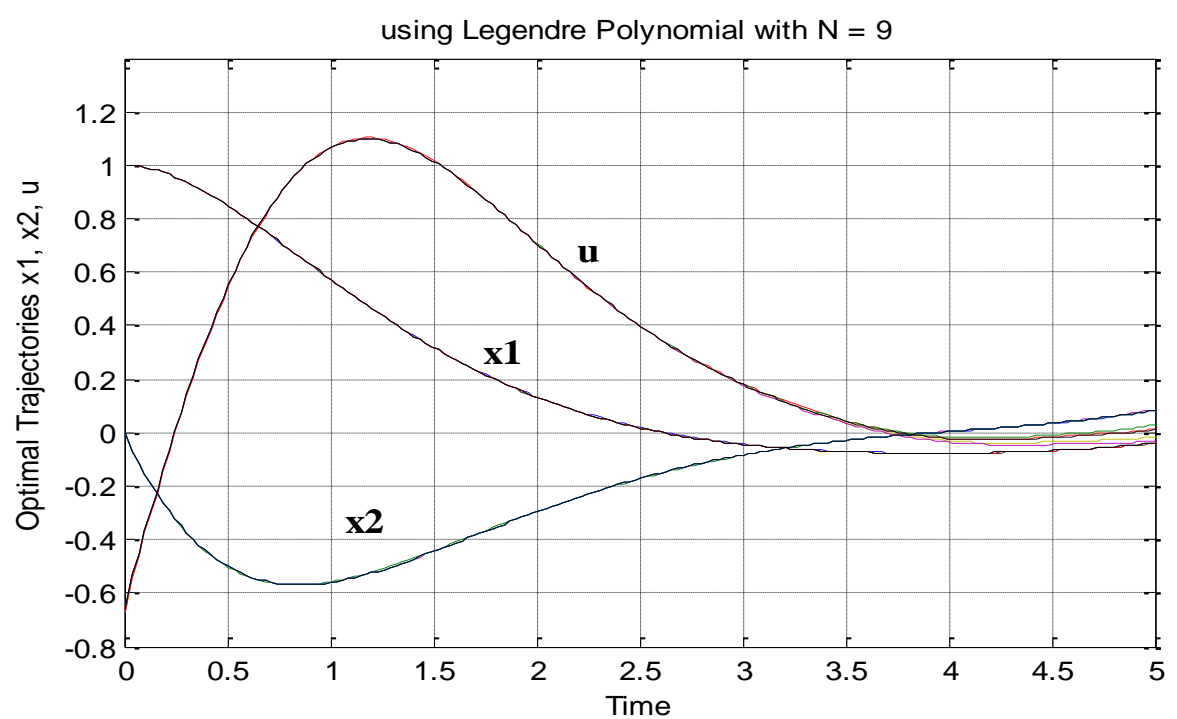

Figure 1. Optimal trajectories of Van der Pol problem using Legendre polynomials

Table 2. values of $\boldsymbol{J}$ obtained using other methods

\begin{tabular}{|l|c|l|}
\hline \multicolumn{1}{|c|}{ Source } & \multicolumn{1}{c|}{ J } & \multicolumn{1}{c|}{ Method } \\
\hline Jaddu [10] & 1.433487 & Quasilinearization and state parameterization \\
\hline Bullock and Franklin [19] & 1.433508 & Second variation \\
\hline Bashein and Enns [20] & 1.438097 & Quasilinearization and discretization \\
\hline
\end{tabular}

\section{Conclusion}

This paper presents a numerical method for solving a class of nonlinear optimal control problems. This method is based on combining the Banks iterative technique with state parameterization using Legendre polynomials to convert the original nonlinear optimal control problem into a sequence of quadratic programming ones. To show the effectiveness of the method, the Van der Pol oscillator problem is solved and the simulation results obtained indicate that the method gives a good and comparable results with other methods. 


\section{References}

[1] M. Tomas-Rodriguez and S. P. Banks, "Linear approximations to nonlinear dynamical systems with applications to stability and spectral theory", IMA Journal of Control and Information, vol. 20, no. 1, (2003), pp. 89-103.

[2] S. P. Banks and K. Dinesh, "Approximate optimal control and stability of nonlinear finite and infinitedimensional systems", Annals of Operations Research, vol. 98, (2000), pp. 19-44.

[3] M. Tomas-Rodriguez and S. P. Banks, "An iterative approach to eigenvalue assignment for nonlinear systems", Proceedings of the 45th IEEE Conference on Decision \& Control, (2006), pp. 977-982.

[4] C. N. Hernandez, S. P. Banks and M. Aldeen, "Observer Design for Nonlinear Systems using Linear Approximations", IMA Journal of Mathematical Control and Information, vol. 20, no. 3, (2003), pp. 359370.

[5] M. Tomas-Rodriguez, S. P. Banks and M. U. Salamci, "Sliding Mode Control for Nonlinear Systems: An Iterative Approach", Proceedings of the 45th IEEE Conference on Decision \& Control, (2006), pp. 49634968.

[6] C. Buskens and H. Maurer, "SQP method for solving optimal control problems with control and state constraints: adjoint variables, sensitivity analysis and real time control", Journal of Computational and Applied Mathematics, vol. 120, no. 1, (2000), pp. 85-108.

[7] J. T. Betts, "Practical Methods for Optimal Control using Nonlinear programming", SIAM, Philadelphia, (2001).

[8] D. Kraft, "On converting optimal control problems into nonlinear programming problems", Computational Mathematical Programming, vol. 15, Ed. Schittkowski, Springer, Berlin, (1985), pp. 261-280.

[9] J. Betts, "Issues in the direct transcription of optimal control problem to sparse nonlinear programs", Computational Optimal Control, Ed. R. Bulirsch and D. Kraft, Birkhauser, Germany, (1994), pp. 3-17.

[10] H. Jaddu, "Numerical methods for solving optimal control problems using Chebyshev polynomials", PHD Thesis, (1998).

[11] H. Jaddu, "Direct solution of nonlinear optimal control problems using quasilinearization and Chebyshev polynomials", Journal of the Franklin Institute, vol. 339, no. 4, (2002), pp. 479-498.

[12] H. Jaddu and M. Vlach, "Successive approximation method for non-linear optimal control problems with applications to a container crane problem", Optimal Control Applications and Methods, vol. 23, no. 5, (2002), pp. 275-288.

[13] C. J. Goh and K. L. Teo, "Control parameterization: A unified approach to optimal control problems with general constraints", Automatica, vol. 24, no. 1, (1988), pp. 3-18.

[14] J. Vlassenbroeck and R. Van Doreen, "A Chebyshev technique for solving nonlinear optimal control problems", IEEE Transactions on Automatic Control, vol. 33, no. 4, (1988), pp. 333-340.

[15] P. A. Frick and D. J. Stech, "Epsilon-Ritz method for solving optimal control problems: Useful parallel solution method", Journal of Optimization Theory and Applications, vol. 79, no. 1, (1993), pp. 31-58.

[16] R. Bellman and R. Kalaba, "Quasilnearization and Nonlinear Boundary Value Problems", Elsevier, New York, (1965).

[17] W. W. Bell, "Special Functions For Scientist and Engineers", D. Van Nostrand Company, London, (1968).

[18] I.S. Gradshteyn and I.M. Ryzhik, "Table of Integrals, Series and Products", 7th edition, ed. Alan Jeffrey and Daniel Zwillinger, Elsevier, (2007)

[19] T. Bullock and G. Franklin, "A second order feedback method for optimal control computations", IEEE Transactions on Automatic Control, vol. 12, no. 6, (1967), pp. 666- 673.

[20] G. Bashein and M. Enns, "Computation of optimal control by a method combining quasi-linearization and quadratic programming", International Journal of Control, vol. 16, no. 1, (1972), pp. 177-187. 


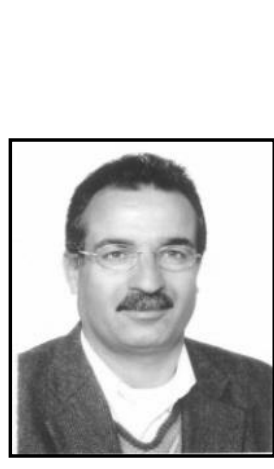

\section{Authors}

Hussein Jaddu received his BSc. in Electrical Engineering from Bir Zeit University, Palestine, in 1987, MSc. in Electronics (digital systems) from Hatfield Polytechnic in England 1991, $\mathrm{PhD}$ in information Systems from Japan Advanced Institute of Science and Technology JAIST, Japan 1998. He worked as a research associate at JAIST during the period 2000-2002. He is with Electronics Engineering Department of Al Quds University in Palestine where he works as an associate professor. In addition to the academic position he also serve as the university vice president for administrative and financial affairs. His main areas of research interests are computational of optimal control, wavelets, congestion control, obstacle avoidance.

Amjad Majdalawi received his BSc. in Electronics Engineering from Yarmouk University, Jordan, in 1997. He then worked in several private sector factories as an electrical/control engineer. In 2010, he received his MSc. degree in electronics and computer engineering from Al Quds University. He is currently working at the ministry of education and higher education in Palestine in the technical education, His main areas of research interests are computational of optimal control, digital communication, and SCADA systems. 
International Journal of Control and Automation Vol.7, No.3 (2014) 